\title{
Observaciones finales: perspectivas para futuras investigaciones sobre gobernanza y la reforma del sector seguridad
}

https://doi.org/10.21830/9789585350601.07

\author{
Martha Lucia Bahamón Jaral \\ Wisman Johan Diaz Castillo \\ Ximena A. Cujabante Villamiß \\ Alex Camilo Durán Montaño ${ }^{4}$ \\ Mateo Morales Callejas \\ Universidad Militar Nueva Granada \\ Manuel Alexánder Betancur Montoya ${ }^{6}$ \\ Escuela Militar de Cadetes "General José María Córdova"
}

1 Abogada de la Universidad Libre de Colombia, especialista en Derecho Administrativo de la Universidad Santo Tomás y magíster en Defensa de los Derechos Humanos y Derecho Internacional Humanitario. Exvicerrectora Académica de la Universidad Militar Nueva Granada en el periodo 20092015. Directora de Posgrados de la Facultad de Relaciones Internacionales, Estrategia y Seguridad. Docente tiempo completo de carrera. Asesora y consultora de instituciones de educación superior. OrCID: https:/orcid.org/0000-0002-5877-6886. Contacto: martha.bahamon@unimilitar.edu.co

2 Profesional en Derecho de la Fundación Universitaria Agraria de Colombia, especialista en Derecho Administrativo de la Universidad Santo Tomás. Materias finalizadas en la Maestría en Derecho Administrativo de la Universidad Santo Tomás. Orcid: https://orcid.org/0000-0001-6370-1945. Contacto: wisman.diaz@uniagraria.edu.co

3 Politóloga de la Universidad Javeriana, especialista en Negociación y Relaciones Internacionales de la Universidad de los Andes, magíster en Asuntos Internacionales y doctora en Estudios Políticos de la Universidad Externado de Colombia. Docente de tiempo completo de la Facultad de Relaciones Internacionales, Estrategia y Seguridad de la Universidad Militar Nueva Granada. OrcID: https:/orcid. org/0000-0002-5473-163X. Contacto: ximena.cujabante@unimilitar.edu.co

4 Politólogo de la Universidad de los Andes. Investigador de la Universidad Militar Nueva Granada. Orcid: https://orcid.org/0000-0001-9701-1520. Contacto: ac.duran1035@uniandes.edu.co

5 Politólogo y magíster en Ciencia Política de la Universidad de los Andes. Orcid: https://orcid. org/0000-0001-5272-1959.

6 Magister en Estudios Políticos e Internacionales, Universidad del Rosario. Profesional en Relaciones Internacionales y Estudios Políticos, Universidad Militar Nueva Granada. Profesional en Ciencias Militares, Escuela Militar de Cadetes “General José María Córdova”. Orcid: https://orcid.org/0000-00016511-9000 
El primer capítulo establece que se empezó a formar una agenda de acción transnacional a partir de la noción de control democrático de las Fuerzas Armadas y la división de los actores sujetos de estos procesos en un núcleo de fuerzas de seguridad, los cuerpos de administración y supervisión de dichas fuerzas y otras fuerzas no-estatutarias o no-estatales. La comunidad internacional de donantes y operadores de seguridad formaron una agenda programática para consolidar normas democráticas que rijan a las fuerzas de seguridad con los principios de la buena gobernanza y el imperio de la ley, y también la vincularon a otros temas neurálgicos de la agenda internacional como la justicia, el desarrollo y la igualdad de género. En medio de este ejercicio, se esclareció que la reforma del sector seguridad aplica a programas, políticas, iniciativas y actividades concretas que buscan fortalecer el control democrático civil de las Fuerzas Armadas en contextos de transición política, mientras que la gobernanza del sector seguridad es la plataforma de principios en los que deben fundamentarse dichos programas, políticas, iniciativas y actividades.

Por su parte, la academia introdujo a la discusión sobre gobernanza y reforma del sector seguridad un entendimiento más amplio de seguridad, aludiendo a múltiples conceptos nuevos de seguridad como seguridad humana, comprehensiva, extendida, colectiva e interconectada. Adicionalmente, pensadores provenientes de la academia ilustraron cómo los procesos de reforma del sector seguridad deben articularse con normas regionales y globales, cuáles son exactamente los principios de gobernanza que deben regir, y las conexiones entre la gobernanza y la reforma del sector seguridad y la reforma de las relaciones cívico-militares.

Asimismo, el capítulo muestra cómo la producción académica sobre gobernanza y reforma del sector seguridad ha expandido en los últimos quince años el entendimiento y la discusión sobre los nexos que la gobernanza y la reforma del sector seguridad tienen con conceptos como seguridad humana, desarrollo, justicia, construcción de Estado y apropiación local. De igual forma, evidencia que se han refinado las líneas de intersección entre la reforma del sector seguridad y los elementos clave de la reforma democrática de las relaciones cívico-militares. No obstante, la literatura se centra cada vez más en casos prácticos específicos de procesos de reforma del sector seguridad, 
los desafíos prácticos de formular y ejecutar procesos integrales de reforma del sector seguridad y, adicionalmente, en definir criterios claros para evaluar procesos de reforma del sector seguridad a nivel mundial.

Como se puede apreciar en el segundo capítulo, es indudable que Colombia está atravesando un periodo clave para revisar su sector de seguridad y defensa con miras en el posacuerdo o en la estabilización del territorio (según se mire). La fuerza pública del país ha sido pionera en muchos aspectos que atañen a los procesos de profesionalización, modernización, transformación y ahora reforma. Las mismas dinámicas del conflicto han incentivado a las instituciones a adaptarse a las mutaciones de una guerra que ha involucrado a un sinnúmero de actores e intereses políticos y económicos. Ante la falta de una clara dirección desde el poder civil, las fuerzas de seguridad y defensa han debido llevar sus procesos de manera autónoma en respuesta a los importantes retos de seguridad que ha debido enfrentar el país.

Estas condiciones han hecho que los procesos de reforma en diferentes frentes sean casos bastante atípicos en comparación con aquellos usualmente referenciados por la literatura académica, ya sea porque no se dieron al finalizar el conflicto, o porque han involucrado otros componentes, como lo internacional, en los momentos y contenidos de las reformas. Muy seguramente, gran parte de la explicación tiene que ver precisamente con el desarrollo tan particular y complejo de nuestro conflicto armado.

Pese a que los cambios se han dado en el interior de las instituciones de seguridad y defensa, y el futuro cercano sugiere que esto se va a seguir presentando, sobre todo a razón del vaivén de los gobiernos de turno, no deja de ser preocupante que la reforma del sector de seguridad y defensa no sea ya un tema de debate público encabezado desde el Ministerio de Defensa y no únicamente parte de discusiones académicas cerradas que no trascienden al debate nacional. Como se mencionó anteriormente, se hace necesario contar con una guía civil en los horizontes de seguridad y defensa, de manera tal que existan directrices de Estado y no únicamente de gobierno. Generar una ley de seguridad y defensa es sin duda clave en todo este debate, como se desarrolló en el cuarto capítulo. La falta de direccionamiento respecto hacia dónde y cómo transformarse en relación con las necesidades del país puede conducir al final a que cada fuerza (Policía y Fuerzas Militares) terminen por funcionar 
como ruedas sueltas, que se solapan en el desarrollo de ciertas funciones y que generan enormes sobrecargas en términos de roles y acciones que no deben ser desarrollados por este tipo de instituciones. Por más profesionales, preparadas y sofisticadas que sean las fuerzas de seguridad de un país, nunca van a lograr suplir las funciones del Estado.

Los casos analizados en el segundo capítulo, por ejemplo, ponen de presente diferentes problemáticas que se agudizan por la falta de este direccionamiento civil. Con respecto a los Derechos Humanos, ya se ha comentado cómo todo el proceso de inclusión de las normativas por parte de las fuerzas fue traumático, pues si bien estas fueron adoptadas y ratificadas por los gobiernos del momento, la transferencia a las fuerzas de seguridad fue mucho más complicada. Gran parte del problema puede tener que ver, precisamente, con el hecho de que, hasta ese momento y a razón de la filosofía generada por la doctrina Lleras, los militares y policías hacían lo que tenían que hacer para mantener la seguridad y el orden público. Con razón, Leal ha hablado de una "seguridad nacional a la deriva". Los procesos de socialización no fueron planeados ni estructurados, sino que se fueron dando de acuerdo con la necesidad de contar con las ayudas provenientes de Estados Unidos (en su gran mayoría).

Hoy en día, como se sugirió, las normativas de Derechos Humanos forman parte de la doctrina militar y policial, pero aún está por verse hasta qué punto se ha hecho un seguimiento sistemático para verificar que esta adopción corresponde con una interiorización real. La carencia de una guía en materia de seguridad y defensa agrava esta situación, en la medida en que favorece el surgimiento de estrategias ad hoc en aras de generar impacto mediático que no solo pueden terminar afectando a las fuerzas de seguridad, sino también minar, realmente, el cumplimiento del respeto a los Derechos Humanos. Basta con recordar el reciente caso en el cual The New York Times afirmaba que las autoridades militares habían solicitado a las unidades militares duplicar los resultados en 2019, lo que claramente podría traer a escena nuevos casos de los mal llamados "falsos positivos".

Frente al tema de género, es importante señalar que los avances que ha habido deben ser resaltados y evidenciados positivamente. La política de 
transversalización es sin duda un paso hacia delante en materia de reforma del sector seguridad y defensa y se debe ahondar su desarrollo. Si embargo, algo similar ocurre frente a este respecto. La ausencia de directivas claras en cuanto hacia dónde y cómo desarrollar la seguridad y la defensa del país conduce a que desarrollos como este se vean seriamente truncados. Si bien la política existe, los resultados no solo son desiguales entre las fuerzas (siendo la Policía la que más la ha desarrollado), sino que corren el riesgo de frenarse del todo.

Como se mencionó, gran parte de lo que se ha hecho en materia de desarrollo del enfoque de género (y no de inclusión de las mujeres) se debe a los insumos generados por la cooperación internacional, especialmente del fondo multidonante de Naciones Unidas. Para los funcionarios policiales que trabajan en estos proyectos es un hecho que sin la voluntad ni el apoyo de estos donantes no se hubiera podido avanzar en la transversalización del enfoque de género, ya que el Ministerio de Defensa no destina rubros constantes para generar acciones en función de esta meta. Por supuesto, esta situación se agrava en la medida en que los temas "duros" de seguridad sigan siendo priorizados y a los asuntos "blandos" solo se les asignen recursos cuando los demás temas de seguridad marchan como es debido. En este sentido, es necesario capitalizar los avances que se han tenido en la materia, poner a dialogar en espacios formales a las fuerzas para compartir sus experiencias y aprender de las mejores prácticas.

Finalmente, vale la pena sugerir que si bien la estrategia de cooperación en seguridad ha sufrido un importante proceso de institucionalización (formal e informal) — lo que se aprecia por ejemplo revisando los documentos de política de defensa y seguridad de los últimos gobiernos_-, es importante clarificar el para qué. Como estrategia, la reducción del pie de fuerza sin duda no es exitosa, en la medida en que no ha generado, como tal, envíos de personal a misiones internacionales, por cuanto gran parte de la cooperación que ofrece el país se hace por medio de asistencia técnica que no implica un intercambio permanente de personal.

Indudablemente, la estrategia de exportación de seguridad ha posicionado a Colombia como un referente en la materia y por lo tanto como un jugador activo en el escenario regional. Sin embargo, no quedan claros cuáles son los objetivos a largo plazo que se persiguen, ni cómo se pueden capitalizar los 
resultados de estos procesos de asistencia e intercambio para reformar el sector seguridad del país. De nuevo, es necesario contar con un direccionamiento claro en el quehacer internacional de las fuerzas de seguridad y defensa para mantener al día la profesionalización de estas instituciones, sobre todo teniendo en cuenta los importantes retos de seguridad que aún se deben enfrentar.

En cuanto al tercer capítulo, casos como los de Suma ponen de presente dos importantes puntos de cara a la construcción de paz y el escenario actual de Colombia. Por una parte, que pese a la situación de orden público es posible volcar la Policía hacia tareas que caen dentro de su misión institucional. Esto no está desconectado del orden público, como a primera vista se podría pensar. De hecho, es todo lo contrario, pues generar e implementar metodologías que acercan la Policía y a la comunidad por medio de un relacionamiento genuino permite crear unas condiciones propicias para desactivar posibles focos de violencia, así como desincentivar la presencia de actores armados ilegales en los territorios, en la medida en que habrá un tejido social fuerte, comunicación y confianza entre los actores locales.

Por otra parte, este tipo de experiencias permite entender que hacer paz territorial, en lo que atañe a la seguridad, es posible. Por supuesto, para que sea operativo a nivel país se requiere una serie de cambios estructurales que van desde el financiamiento de los fondos de seguridad, hasta los incentivos e indicadores utilizados para medir el desempeño policial. Esto no es poca cosa, si se entiende que gran parte del problema radica en que la Policía forma parte del Ministerio de Defensa. Igualmente, para la adopción efectiva de estas metodologías se requiere un cambio sustancial en la formación policial, como se sugirió más arriba. No solo en términos de formar policías aptos para materializar aproximaciones que requieren de capacidades blandas, sino también en términos de desmantelar esas lógicas internas de la institución que siguen generando una división artificial entre problemas "fuertes" y "débiles" de seguridad, que lo que hace es profundizar el carácter militarista de la Policía.

Finalmente, vale la pena cerrar sugiriendo que este tipo de experiencias le ofrecen al país la oportunidad de apropiarse de las reformas al sector seguridad. El caso de Suma, como se vio, es una oportunidad que fue posible gracias a la cooperación internacional y al entusiasmo de un grupo de policías que activa- 
mente buscaron el financiamiento necesario para implementar el Modelo de Construcción de Paz. El Estado colombiano está en deuda de prestar atención a estas iniciativas, planificadas e implementadas de manera endógena en la institución. Así, podrá proyectar la hoja de ruta que debería adquirir la reforma del sector seguridad en el país para enfrentar los retos que plantea un escenario de posacuerdo y no desaprovechar las oportunidades que vienen con este. Hay un riesgo enorme de que estas acciones de las instituciones caigan en saco roto al no tener respuesta de la autoridad civil correspondiente y, por lo tanto, se desaprovechen los recursos inyectados por medio de la cooperación internacional, así como apagar las voces progresistas en sectores tan conservadores como la seguridad y la defensa.

Entre las principales conclusiones del análisis que propone el cuarto capítulo se encuentra la necesidad de consolidar un componente normativo claro en materia de seguridad y defensa nacional. Se trata de un asunto de gran envergadura para el caso colombiano, pues con ello no solo se logra apoyar el alcance y la protección de los intereses nacionales, sino también el uso adecuado de las capacidades del Estado. De esta manera, ante la necesidad de determinar el contenido normativo para el caso de Colombia, resulta oportuno analizar y definir los componentes que son relevantes para los países analizados, los cuales cuentan actualmente con este instrumento jurídico.

La selección de los países se realizó de acuerdo con tres características relevantes para Colombia: (1) en su desarrollo como nación debían haber atravesado por procesos de transición que hubieran incidido en ese contenido normativo; (2) las Fuerzas Militares debían tener importancia en su formación como nación, pues en el caso colombiano estas han tenido una participación activa en comparación con otros países, y (3) debían contar con un contenido normativo claro en seguridad y defensa. Asimismo, el análisis incluyó las circunstancias que determinaron la degradación del entorno en seguridad y defensa de Estados centroamericanos que atravesaron procesos de transición luego de firmar acuerdos de paz.

Como se evidenció con el estudio de los casos, la base de una ley de seguridad y defensa $\longrightarrow$ o de otros instrumentos que regulen la materia, como los libros blancos de defensa- consiste en establecer los fundamentos consti- 
tucionales que definan la responsabilidad del Estado en la administración de este ámbito, ya sea entendido como un derecho, un servicio o una función. Igualmente, queda claro que no se trata únicamente de una responsabilidad de gobierno, sino que involucra a todas las instituciones del Estado e, inclusive, al conjunto de la sociedad.

Para el caso colombiano, uno de los aspectos relevantes que se pueden sustraer del análisis es la comprensión de la seguridad como un elemento de todo el Estado, de tal manera que cada uno de los actores debe participar en su consolidación. Esto se materializa respecto de la claridad jurídica de aquellos fines que sean considerados como intereses de toda la nación, sobre los cuales deben orbitar todas las acciones del Estado y la sociedad. Asimismo, se hace oportuno que este instrumento integre las distintas capacidades del Estado y no solo el componente militar, sino además cada una de las instituciones que contribuyen a alcanzar estos intereses nacionales.

Otro elemento que puede contribuir a que Colombia consolide un instrumento jurídico que le permita regular efectivamente la seguridad y la defensa nacional es determinar con claridad la relevancia de la inversión social para las regiones que fueron afectadas por el conflicto armado. De acuerdo con las lecciones aprendidas de los países centroamericanos, la seguridad en el territorio no se logra solamente por medio de la fuerza pública, sino que también se necesita toda la institucionalidad.

Dentro de estos mismos aportes, se puede destacar la formación de la diplomacia colombiana con fortalezas representativas y un dinamismo mayor dentro del sistema internacional, lo cual permite evidenciar una nueva herramienta que también contribuya a consolidar los intereses nacionales del Estado. En este sentido, se hace indispensable generar una integración clara entre la política exterior colombiana y la seguridad y defensa del país — en cuanto herramientas que permiten lograr estos intereses—, así como contar con una proyección que sea reconocida y desarrollada por el instrumento jurídico.

Asimismo, se hace necesario que la ley de seguridad y defensa nacional defina y establezca las siguientes consideraciones: (1) definir lo que significa para el país la seguridad y la defensa; (2) identificar las amenazas; (3) integrar el instrumento normativo con la política exterior del país; (4) establecer los 
medios y las capacidades del Estado para garantizar la seguridad y la defensa; (5) definir las condiciones en que se debe ejercer el control territorial; (6) determinar todos los campos de acción del Estado en favor de la seguridad y defensa nacional, y (7) fomentar la innovación desde la academia en favor de la seguridad y la defensa nacional. Asimismo, es indispensable que este contenido normativo evite (1) la falta de transformación institucional, (2) la falta de control territorial por el Estado y (3) la falta de participación de todos los actores de los conflictos. Finalmente, una lección aprendida muy importante es que esta ley divida claramente las funciones sobre seguridad y defensa entre las instituciones de la Fuerza Pública.

Por su parte, el quinto capítulo arroja varias conclusiones. En primer lugar, el contexto histórico de los casos evidencia los peligros que conlleva la centralidad de las Fuerzas Armadas como un actor político y, simultáneamente, la extralimitación de sus funciones "tradicionales". Para contribuir a la creación de una nueva estructura de gobernanza del sector seguridad en el marco del posconflicto en Colombia, parecería esencial definir claramente los roles entre la Policía Nacional y las Fuerzas Armadas, de tal manera que se revierta el statu quo en el cual ha existido una Policía Nacional militarizada y unas Fuerzas Armadas desempeñando tareas de mantenimiento del orden público.

Los fracasos en materia de efectividad en los casos seleccionados ofrecen lecciones importantes. Estos hallazgos marcan un derrotero claro para crear una nueva estructura de gobernanza del sector en Colombia orientado principalmente hacia:

- Una formulación de objetivos claros y delimitados con un proceso de operacionalización y aterrizaje claro por parte de tomadores de decisión y reformadores civiles a cargo.

- Un marco normativo coherente con el objetivo central de establecer un mayor grado de control democrático civil sobre las Fuerzas Armadas.

- Una concepción de seguridad en la cual el pie de fuerza policial y militar sea un instrumento dentro de una oferta institucional amplia orientada a reducir las condiciones estructurales del conflicto y, 
simultáneamente, aumentar la capacidad de gestión de conflictividades por parte de autoridades civiles.

Estos casos también presentaron obstáculos políticos, culturales y normativos al establecimiento del control democrático civil sobre las Fuerzas Armadas, además de imperativos políticos derivados de condiciones deterioradas de seguridad ciudadana. Específicamente, frente al caso colombiano y los prospectos de una nueva estructura de gobernanza del sector seguridad, es necesario:

- Identificar los obstáculos políticos y culturales en el interior de las Fuerzas Armadas frente a medidas que busquen establecer un mayor control democrático civil de estas.

- Contrarrestar las prerrogativas institucionales para las Fuerzas Armadas que puedan derivarse del marco normativo y los esquemas de justicia transicional.

- Aumentar la autoridad política civil y su incidencia en la formulación e implementación de políticas de seguridad ciudadana para que futuros desarrollos en índices de percepción y confianza institucional no justifiquen la implementación de políticas militaristas o de "mano dura".

Los tres casos dejan mucho que desear respecto a las reformas de justicia y desarrollo que deben acompañar la reforma del sector seguridad. En Colombia, es esencial que una nueva estructura de gobernanza del sector seguridad garantice la independencia de la rama judicial frente al establecimiento militar, que reduzca la incidencia de mecanismos paralelos de justicia militar sobre casos de violaciones de Derechos Humanos o Derecho Internacional Humanitario, y que se implementen medidas para reducir la impunidad o la falta de esclarecimiento frente a episodios de violaciones de Derechos Humanos o crimen violento.

Mientras que Perú, Chile y Guatemala ofrecen lecciones por sus falencias en materia de apoyo social y apropiación local, el caso salvadoreño ilustra las ventajas de tener un proceso inclusivo (si bien su resultado final no es efectivo). Para la nueva estructura de gobernanza del sector seguridad que se pretende construir en Colombia durante los próximos años es esencial incluir de manera 
activa, pública y participativa a secciones representativas de la sociedad civil para garantizar la apropiación y apoyo frente a un proceso que, por su inclusión, se considerará legítimo.

Asimismo, los tres casos seleccionados ofrecen lecciones sobre los peligros de tolerar prerrogativas económicas amplias para las Fuerzas Armadas y la corrupción endémica en sus filas y eslabones superiores. Para Colombia, es fundamental que la construcción de un nuevo marco de gobernanza del sector seguridad identifique y repliegue las prerrogativas económicas de las Fuerzas Armadas que no son esenciales para el desempeño de labores de defensa características de la redefinición de roles que debe acompañar la reforma. De igual modo, es fundamental que este proceso sea acompañado de inversión pública en áreas claves como el empleo, la salud y la satisfacción de necesidades básicas, de tal manera que el pie de fuerza policial y militar sean instrumentos dentro de una oferta institucional amplia para mejorar las condiciones de seguridad.

Tanto el proceso peruano como el chileno se caracterizaron por su hermetismo y participación casi nula de la sociedad internacional, al igual que el caso guatemalteco, mientras que en El Salvador la participación activa de la sociedad internacional mediante acción multilateral liderada por la $\mathrm{ONU}_{\mathrm{NU}}$ probó ser fundamental para legitimar el proceso de reforma del sector seguridad, aunque sus resultados hayan sido deficientes. Como lección para el caso colombiano y el objetivo de crear una nueva estructura de gobernanza del sector seguridad, es imprescindible la participación de la sociedad internacional para ejercer una veeduría sobre el proceso y generar alertas tempranas sobre desarrollos normativos o procesos políticos que puedan crear eventuales fracasos en el propósito de instaurar un control democrático civil sobre las Fuerzas Armadas, o cualquiera de las otras variables analizadas. 